\title{
More on contra $\delta$-precontinuous functions
}

\author{
Miguel Caldas, Saeid Jafari, Takashi Noiri, and \\ Marilda Simões
}




\title{
MORE ON CONTRA $\delta$-PRECONTINUOUS FUNCTIONS
}

\author{
MIGUEL CALDAS, SAEID JAFARI, TAKASHI NOIRI, AND MARILDA SIMÕES
}

Received 3 June, 2006

\begin{abstract}
In [4], Dontchev introduced and investigated a new notion of continuity called contracontinuity. Recently, Jafari and Noiri [8-10] introduced new generalizations of contra-continuity called contra- $\alpha$-continuity, contra-super-continuity and contra-precontinuity. Recently, Ekici and Noiri [6] have introduced a new class of continuity called contra $\delta$-precontinuity as a generalization of contra-continuity. In this paper, we obtain some more properties of contra $\delta$-precontinuous functions.
\end{abstract}

2000 Mathematics Subject Classification: 54C10, 54D10

Keywords: topological spaces, $\delta$-preopen sets, preclosed sets, contra-continuous functions, contra $\delta$-precontinuous functions

\section{INTRODUCTION AND PRELIMINARIES}

Recently, Jafari and Noiri have introduced and investigated the notions of contraprecontinuity [10], contra- $\alpha$-continuity [9] and contra-super-continuity [8] as a continuation of research done by Dontchev [4] and Dontchev and Noiri [5] on the interesting notions of contra-continuity and contra-semi-continuity, respectively. Caldas and Jafari [3] introduced and investigated the notion of contra- $\beta$-continuous functions in topological spaces. Raychaudhuri and Mukherjee [15] introduced the notions of $\delta$-preopen sets and $\delta$-almost continuity in topological spaces. The class of $\delta$-preopen sets is larger than one of preopen sets. Recently, by using $\delta$-preopen sets, Ekici and Noiri [6] have introduced the notion of contra $\delta$-precontinuity as a generalization of contra-precontinuity.

In this paper, we obtain the further properties of contra $\delta$-precontinuous functions. Throughout this paper, all spaces $(X, \tau)$ and $(Y, \sigma)$ (or $X$ and $Y$ ) are topological spaces. A subset $A$ of $X$ is said to be regular open (resp., regular closed) if $A=\operatorname{Int}(\mathrm{Cl}(A))$ (resp., $A=\mathrm{Cl}(\operatorname{Int}(A))$ ) where $\mathrm{Cl}(A)$ and $\operatorname{Int}(A)$ denote the closure and interior of $A$. A subset $A$ of a space $X$ is called preopen [12] (resp., semi-open [11], $\alpha$-open [14], $\beta$-open [1]) if $A \subset \operatorname{Int}(\mathrm{Cl}(A))$ (resp., $A \subset \mathrm{Cl}(\operatorname{Int}(A))$, $A \subset \operatorname{Int}(\mathrm{Cl}(\operatorname{Int}(A))), A \subset \mathrm{Cl}(\operatorname{Int}(\mathrm{Cl}(A))))$. The complement of a preopen (resp., semi-open, $\alpha$-open, $\beta$-open) set is said to be preclosed (resp., semi-closed, $\alpha$-closed, $\beta$-closed). The collection of all closed (resp., semi-open, clopen) subsets of $X$ will 
be denoted by $C(X)$ (resp., $S O(X), C O(X))$. We set $C(X, x)=\{V \in C(X) \mid x \in V\}$ for $x \in X$. We define $C O(X, x)$ in a similar way.

The notion of the $\delta$-closure of $A$ which is denoted by $\delta \mathrm{Cl}(A)$ was introduced by Veličko [19] and is widely investigated in the literature. The $\delta$-closure of $A$ is the set $\{x \in X \mid \operatorname{Int}(\mathrm{Cl}(U)) \cap A \neq \varnothing$ for every open set $U$ containing $x\}$. If $\delta \mathrm{Cl}(A)=A$, then $A$ is said to be $\delta$-closed [19]. The complement of a $\delta$-closed set is said to be $\delta$ open. The union of all $\delta$-open sets contained in $A$ is called the $\delta$-interior of $A$ and is denoted by $\delta \operatorname{Int}(A)$. A subset $A$ of a topological space $X$ is said to be $\delta$-preopen [15] if $A \subset \operatorname{Int}(\delta \mathrm{Cl}(A))$. The complement of a $\delta$-preopen se is said to be $\delta$-preclosed. The intersection (union) of all $\delta$-preclosed ( $\delta$-preopen) sets containing (contained in) $A$ in $X$ is called the $\delta$-preclosure ( $\delta$-preinterior) of $A$ and is denoted by $\delta \mathrm{Cl}_{p}(A)$ (resp., $\left.\delta \operatorname{Int}_{p}(A)\right)$. By $\delta P O(X)($ resp., $\delta P C(X))$, we denote the collection of all $\delta$-preopen (resp., $\delta$-preclosed) sets of $X$.

Lemma 1 ([2,15,17]). The following properties holds for the $\delta$-preclosure of a set in a space $X$ :

(1) Arbitrary union (intersection) of $\delta$-preopen ( $\delta$-preclosed) sets in $X$ is $\delta$-preopen (resp., $\delta$-preclosed).

(2) $A$ is $\delta$-preclosed in $X$ iff $A=\delta \mathrm{Cl}_{p}(A)$.

(3) $\delta \mathrm{Cl}_{p}(A) \subset \delta \mathrm{Cl}_{p}(B)$ whenever $A \subset B(\subset X)$.

(4) $\delta \mathrm{Cl}_{p}(A)$ is $\delta$-preclosed in $X$.

(5) $\delta \mathrm{Cl}_{p}\left(\delta \mathrm{Cl}_{p}(A)\right)=\delta \mathrm{Cl}_{p}(A)$.

(6) $\delta \mathrm{Cl}_{p}(A)=\{x \in X \mid U \cap A \neq \varnothing$ for every $\delta$-preopen set $U$ containing $x\}$.

(7) $\delta \mathrm{Cl}_{p}(A)=A \cup \mathrm{Cl}(\delta \operatorname{Int}(A))$.

(8) If $A$ is $\delta$-open, then $\delta \mathrm{Cl}_{p}(A)=\mathrm{Cl}(A)$.

(9) If $Y \subset X$ is $\delta$-open and $U \in \delta P O(Y)$, then $U \in \delta P O(X)$.

(10) $U \cap V \in \delta P O(U)$ if $U$ is $\delta$-open and $V \in \delta P O(X)$.

Definition 1. A function $f: X \rightarrow Y$ is said to be contra $\delta$-precontinuous [6] (resp., $\delta$-almost continuous [15]) if $f^{-1}(V)$ is $\delta$-preclosed (resp., $\delta$-preopen) in $X$ for each open set $V$ of $Y$.

Definition 2. Let $A$ be a subset of a space $(X, \tau)$. The set $\cap\{U \in \tau \mid A \subset U\}$ is called the kernel of $A[13]$ and is denoted by $\operatorname{ker}(A)$.

Lemma 2 (Jafari and Noiri [8]). The following properties hold for subsets $A, B$ of a space $X$ :

(1) $x \in \operatorname{ker}(A)$ if and only if $A \cap F \neq \varnothing$ for any $F \in C(X, x)$.

(2) $A \subset \operatorname{ker}(A)$ and $A=\operatorname{ker}(A)$ if $A$ is open in $X$.

(3) If $A \subset B$, then $\operatorname{ker}(A) \subset \operatorname{ker}(B)$.

\section{CONTRA $\delta$-PRECONTINUOUS FUNCTIONS}

Theorem 1. The following assertions are equivalent for a function $f: X \rightarrow Y$ : 
(1) $f$ is contra $\delta$-precontinuous.

(2) For every closed subset $F$ of $Y, f^{-1}(F) \in \delta P O(X)$.

(3) For each $x \in X$ and each $F \in C(Y, f(x))$, there exists $U \in \delta P O(X, x)$ such that $f(U) \subset F$.

(4) $f\left(\delta \mathrm{Cl}_{p}(A)\right) \subset \operatorname{ker}(f(A))$ for every subset $A$ of $X$.

(5) $\delta \mathrm{Cl}_{p}\left(f^{-1}(B)\right) \subset f^{-1}(\operatorname{ker}(B))$ for every subset $B$ of $Y$.

Proof. The implications (1) $\Rightarrow(2)$ and (2) $\Rightarrow$ (3) are obvious.

(3) $\Rightarrow(4)$ : Let $A$ be any subset of $X$. Suppose that $y \notin \operatorname{ker}(f(A))$. Then, by Lemma 2, there exists $F \in C(Y, y)$ such that $f(A) \cap F=\varnothing$. For any $x \in f^{-1}(F)$, by (3) there exists $U_{x} \in \delta P O(X, x)$ such that $f\left(U_{x}\right) \subset F$. Hence $f\left(A \cap U_{x}\right) \subset f(A) \cap$ $f\left(U_{x}\right) \subset f(A) \cap F=\varnothing$ and $A \cup U_{x}=\varnothing$. This shows that $x \notin \delta \mathrm{Cl}_{p}(A)$ for any $x \in f^{-1}(F)$. Therefore, $f^{-1}(F) \cap \delta \mathrm{Cl}_{p}(A)=\varnothing$ and hence $F \cap f\left(\delta \mathrm{Cl}_{p}(A)\right)=\varnothing$. Thus, $y \notin f\left(\delta \mathrm{Cl}_{p}(A)\right)$. Consequently, we obtain $f\left(\delta \mathrm{Cl}_{p}(A)\right) \subset \operatorname{ker}(f(A))$.

$(4) \Rightarrow(5)$ : Let B be any subset of $Y$. Them, by (4) and Lemma 2, we have $f\left(\delta \mathrm{Cl}_{p}\left(f^{-1}(B)\right)\right) \subset \operatorname{ker}\left(f\left(f^{-1}(B)\right)\right) \subset \operatorname{ker}(B)$ and therefore $\delta \mathrm{Cl}_{p}\left(f^{-1}(B)\right) \subset$ $f^{-1}(\operatorname{ker}(B))$.

$(5) \Rightarrow(1)$ : Let $V$ be any open set of $Y$. Then, by virtue of Lemma 2, we have $\delta \mathrm{Cl}_{p}\left(f^{-1}(V)\right) \subset f^{-1}(\operatorname{ker}(V))=f^{-1}(V)$ and $\delta \mathrm{Cl}_{p}\left(f^{-1}(V)\right)=f^{-1}(V)$. This shows that $f^{-1}(V)$ is $\delta$-preclosed in $X$.

The following two examples show that $\delta$-almost continuous and contra $\delta$-precontinuous are independent concepts.

Example 1. The identity function on the real line (with the usual topology) is continuous and hence $\delta$-almost continuous but not contra $\delta$-precontinuous, since the preimage of each singleton fails to be $\delta$-preopen.

Example 2. Let $X=\{a, b\}$ be the Sierpinski space endowed with the topology $\tau=\{\varnothing,\{a\}, X\}$. Let $f: X \rightarrow X$ be defined by $f(a)=b$ and $f(b)=a$. Since the inverse image of every open set is $\delta$-preclosed, then $f$ is contra $\delta$-precontinuous, but $f^{-1}(\{a\})$ is not $\delta$-preopen in $(X, \tau)$. Therefore $f$ is not $\delta$-almost continuous.

Definition 3. A function $f: X \rightarrow Y$ is said to be contra-continuous [4] (resp., contra- $\alpha$-continuous [9], contra-precontinuous [10], contra-semi-continuous [5], contra- $\beta$-continuous [3]) if, for each open set $V$ of $Y, f^{-1}(V)$ is closed (resp., $\alpha$-closed, preclosed, semi-closed, $\beta$-closed) in $X$.

For the functions defined above, we have the following implications:

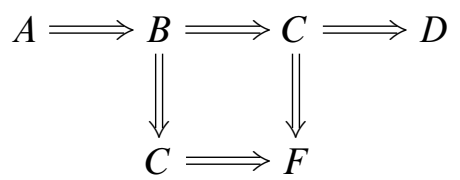


The meaning of symbols here is as follows: $A=$ contra-continuity, $B=$ contra- $\alpha$ continuity, $C=$ contra-precontinuity, $D=$ contra $\delta$-precontinuity, $E=$ contra-semicontinuity, and $F=$ contra- $\beta$-continuity.

It should be mentioned that none of these implications is reversible as shown by the examples stated below.

Example 3 (Jafari and Noiri [9]). Let $X=\{a, b, c\}$. Put $\tau=\{\varnothing,\{a\}, X\}$ and $\sigma=\{\varnothing,\{b\},\{c\},\{b, c\}, X\}$. Then the identity function $f:(X, \tau) \rightarrow(X, \sigma)$ is contra$\alpha$-continuous but not contra-continuous.

Lemma 3 (Caldas et al. [7]). Let $A$ be a subset of $(X, \tau)$. Then the following properties hold:

(1) If $A$ is preopen in $(X, \tau)$, then it is $\delta$-preopen in $(X, \tau)$.

(2) $A$ is $\delta$-preopen in $(X, \tau)$ if and only if it is preopen in $\left(X, \tau_{s}\right)$.

(3) $A$ is $\delta$-preclosed in $(X, \tau)$ if and only if it is preclosed in $\left(X, \tau_{s}\right)$.

Since $\mathrm{Cl}(A) \subset \delta \mathrm{Cl}(A)$ for any subset $A$ of $X$, therefore, every contra-precontinuous is contra- $\delta$-precontinuous but not conversely as following example shows.

Example 4 ([5]). A contra-semi-continuous function need not be contra-precontinuous. Let $f: R \rightarrow R$ be the function $f(x)=[x]$, where $[x]$ is the Gaussian symbol. If $V$ is a closed subset of the real line, its preimage $U=f^{-1}(V)$ is the union of the intervals of the form $[n, n+1], n \in Z$; hence $U$ is semi-open being union of semiopen sets. But $f$ is not contra-precontinuous, because $f^{-1}(0.5,1.5)=[1,2)$ is not preclosed in $R$.

Example 5 ([5]). A contra-precontinuous function need not be contra-semi-continuous. Let $X=\{a, b\}, \tau=\{\varnothing, X\}$ and $\sigma=\{\varnothing,\{a\}, X\}$. The identity function $f:(X, \tau) \rightarrow(Y, \sigma)$ is contra-precontinuous as only the trivial subsets of $X$ are open in $(X, \tau)$. However, $f^{-1}(\{a\})=\{a\}$ is not semi-closed in $(X, \tau)$; hence $f$ is not contra-semi-continuous.

Example 6 ([6]). Let $R$ be the set of real numbers, $\tau$ be the countable extension topology on $R$, i.e., the topology with subbase $\tau_{1} \cup \tau_{2}$, where $\tau_{1}$ is the Euclidean topology of $R$ and $\tau_{2}$ is the topology of countable complements of $R$, and $\sigma$ be the discrete topology of $R$. Define a function $f:(R, \tau) \rightarrow(R, \sigma)$ as follows: $f(x)=1$ if $x$ is rational, and $f(x)=2$ if $x$ is irrational. Then $f$ is contra $\delta$-precontinuous but not contra- $\beta$-continuous, because $\{1\}$ is closed in $(R, \sigma)$ and $f^{-1}(\{1\})=Q$, where $Q$ is the set of rationals, is not $\beta$-open in $(R, \tau)$.

Example 7 ([3]). Let $X=\{a, b, c\}, \tau=\{\varnothing,\{a\},\{b\},\{a, b\}, X\}$ and $Y=\{p, q\}$, $\sigma=\{\varnothing,\{p\}, Y\}$. Let $f:(X, \tau) \rightarrow(Y, \sigma)$ be defined by $f(a)=p$ and $f(b)=f(c)=$ $q$. Then $f$ is contra- $\beta$-continuous but not contra $\delta$-precontinuous since $f^{-1}(\{q\})=$ $\{b, c\}$ is $\beta$-open but not $\delta$-preopen.

Theorem 2. If a function $f: X \rightarrow Y$ is contra $\delta$-precontinuous and $Y$ is regular, then $f$ is $\delta$-almost continuous. 
Proof. Let $x$ be an arbitrary point of $X$ and $V$ an open set of $Y$ containing $f(x)$. Since $Y$ is regular, there exists an open set $W$ in $Y$ containing $f(x)$ such that $\mathrm{Cl}(W) \subset V$. Since $f$ is contra $\delta$-precontinuous, so by Theorem 1 there exists $U \in$ $\delta P O(X, x)$ such that $f(U) \subset \mathrm{Cl}(W)$. Then $f(U) \subset \mathrm{Cl}(W) \subset V$. Hence, $f$ is $\delta$ almost continuous.

The converse of Theorem 2 is not true. Example 1 shows that $\delta$-almost continuity does not necessarily imply contra $\delta$-precontinuity even if the range is regular.

Definition 4. A function $f: X \rightarrow Y$ is said to be:

(1) $(\delta, s)$-preopen if $f(U) \in S O(Y)$ for every $\delta$-preopen set of $X$.

(2) contra- $I(\delta, p)$-continuous if for each $x \in X$ and each $F \in C(Y, f(x))$, there exists $U \in \delta P O(X, x)$ such that $\operatorname{Int}(f(U)) \subset F$.

Theorem 3. If a function $f: X \rightarrow Y$ is contra- $I(\delta, p)$-continuous and $(\delta, s)$-preopen, then $f$ is contra $\delta$-precontinuous.

Proof. Suppose that $x \in X$ and $F \in C(Y, f(x))$. Since $f$ is contra- $I(\delta, p)$-continuous, there exists $U \in \delta P O(X, x)$ such that $\operatorname{Int}(f(U)) \subset F$. By hypothesis $f$ is $(\delta, s)$-preopen, therefore $f(U) \in S O(Y)$ and $f(U) \subset \mathrm{Cl}(\operatorname{Int}(f(U)) \subset F$. This shows that $f$ is contra $\delta$-precontinuous.

Definition 5. A space $(X, \tau)$ is said to be:

(1) locally $(\delta, p)$-indiscrete if every $\delta$-preopen set of $X$ is closed in $X$.

(2) $\delta p$-space if every $\delta$-preopen set of $X$ is open in $X$.

(3) $\delta S$-space if and only if every $\delta$-preopen subset of $X$ is semi-open.

The following theorem follows immediately from Definition 5 .

Theorem 4. If a function $f: X \rightarrow Y$ is contra $\delta$-precontinuous and $X$ is a $\delta S$ space (resp., $\delta p$-space, locally $(\delta, p)$-indiscrete), then $f$ is contra-semi-continuous (resp., contra-continuous, continuous).

Recall that a topological space is said to be:

(1) $(\delta, p)-T_{2}$ ([16]) if for each pair of distinct points $x$ and $y$ in $X$ there exist $U \in \delta P O(X, x)$ and $V \in \delta P O(X, y)$ such that $U \cap V=\varnothing$.

(2) Ultra Hausdorff [18] if for each pair of distinct points $x$ and $y$ in $X$ there exist $U \in C O(X, x)$ and $V \in C O(X, y)$ such that $U \cap V=\varnothing$.

Theorem 5. If $X$ is a topological space and for each pair of distinct points $x_{1}$ and $x_{2}$ in $X$ there exists a map $f$ of $X$ into a Urysohn topological space $Y$ such that $f\left(x_{1}\right) \neq f\left(x_{2}\right)$ and $f$ is contra $\delta$-precontinuous at $x_{1}$ and $x_{2}$, then $X$ is $(\delta, p)-T_{2}$.

Proof. Let $x_{1}$ and $x_{2}$ be any distinct points in $X$. Then by hypothesis, there is a Urysohn space $Y$ and a function $f: X \rightarrow Y$, which satisfies the conditions of the theorem. Let $y_{i}=f\left(x_{i}\right)$ for $i=1,2$. Then $y_{1} \neq y_{2}$. Since $Y$ is Urysohn, there 
exist open neighbourhoods $U_{y_{1}}$ and $U_{y_{2}}$ of $y_{1}$ and $y_{2}$ respectively in $Y$ such that $\mathrm{Cl}\left(U_{y_{1}}\right) \cap \mathrm{Cl}\left(U_{y_{2}}\right)=\varnothing$. Since $f$ is contra $\delta$-precontinuous at $x_{i}$, there exists a $\delta$ preopen neighbourhood $W_{x_{i}}$ of $x_{i}$ in $X$ such that $f\left(W_{x_{i}}\right) \subset \mathrm{Cl}\left(U_{y_{i}}\right)$ for $i=1,2$. Hence we get $W_{x_{1}} \cap W_{x_{2}}=\varnothing$ because $\operatorname{Cl}\left(U_{y_{1}}\right) \cap \mathrm{Cl}\left(U_{y_{2}}\right)=\varnothing$. Then $X$ is $(\delta, p)$ $T_{2}$.

Corollary 1. If $f$ is a contra $\delta$-precontinuous injection of a topological space $X$ into a Urysohn space $Y$, then $X$ is $(\delta, p)-T_{2}$.

Proof. For each pair of distinct points $x_{1}$ and $x_{2}$ in $X, f$ is a contra $\delta$-precontinuous function of $X$ into a Urysohn space $Y$ such that $f\left(x_{1}\right) \neq f\left(x_{2}\right)$ because $f$ is injective. Hence by Theorem $5, X$ is $(\delta, p)-T_{2}$.

Corollary 2. If $f$ is a contra $\delta$-precontinuous injection of a topological space $X$ into a Ultra Hausdorff space $Y$, then $X$ is $(\delta, p)-T_{2}$.

Proof. Let $x_{1}$ and $x_{2}$ be any distinct points in $X$. Then since $f$ is injective and $Y$ is Ultra Hausdorff $f\left(x_{1}\right) \neq f\left(x_{2}\right)$, and there exist $V_{1}, V_{2} \in C O(Y)$ such that $f\left(x_{1}\right) \in$ $V_{1}, f\left(x_{2}\right) \in V_{2}$ and $V_{1} \cap V_{2}=\varnothing$. Then $x_{i} \in f^{-1}\left(V_{i}\right) \in \delta P O(X)$ for $i=1,2$ and $f^{-1}\left(V_{1}\right) \cap f^{-1}\left(V_{2}\right)=\varnothing$. Thus $X$ is $(\delta, p)-T_{2}$.

Lemma 4 ([15]). If $A_{i}$ is a $\delta$-preopen set in a topological space $X_{i}$ for $i=$ $1,2, \ldots, n$, then $A_{1} \times \cdots \times A_{n}$ is also $\delta$-preopen in the product space $X_{1} \times \cdots \times X_{n}$.

Theorem 6. Let $f_{1}: X_{1} \rightarrow Y$ and $f_{2}: X_{2} \rightarrow Y$ be two functions, where

(1) $Y$ is a Urysohn space,

(2) $f_{1}$ and $f_{2}$ are contra $\delta$-precontinuous.

Then the set

$$
\left\{\left(x_{1}, x_{2}\right) \mid f_{1}\left(x_{1}\right)=f_{2}\left(x_{2}\right)\right\}
$$

is $\delta$-preclosed in the product space $X_{1} \times X_{2}$.

Proof. Let $A$ denote the set $\left\{\left(x_{1}, x_{2}\right) \mid f_{1}\left(x_{1}\right)=f_{2}\left(x_{2}\right)\right\}$. In order to show that $A$ is $\delta$-preclosed, we show that $\left(X_{1} \times X_{2}\right) \backslash A$ is $\delta$-preopen. Let $\left(x_{1}, x_{2}\right) \notin A$. Then $f_{1}\left(x_{1}\right) \neq f_{2}\left(x_{2}\right)$. Since $Y$ is Urysohn, there exist open $V_{1}$ and $V_{2}$ of $f_{1}\left(x_{1}\right)$ and $f_{2}\left(x_{2}\right)$ such that $C\left(V_{1}\right) \cap C\left(V_{2}\right)=\varnothing$. Since $f_{i}(i=1,2)$ is contra $\delta$-precontinuous, $f_{i}^{-1}\left(C\left(V_{i}\right)\right)$ is a $\delta$-preopen set containing $x_{i}$ in $X_{i}(i=1,2)$. Hence, by virtue of Lemma 4, $f_{1}^{-1}\left(C\left(V_{1}\right)\right) \times f_{2}^{-1}\left(C\left(V_{2}\right)\right)$ is $\delta$-preopen. Further $\left(x_{1}, x_{2}\right) \in f_{1}^{-1} C\left(V_{1}\right) \times$ $f_{2}^{-1} C\left(V_{2}\right) \subset\left(X_{1} \times X_{2}\right) \backslash A$. It follows that $\left(X_{1} \times X_{2}\right) \backslash A$ is $\delta$-preopen. Thus $A$ is $\delta$-preclosed in the product space $X_{1} \times X_{2}$.

Corollary 3. If $f: X \rightarrow Y$ is contra $\delta$-precontinuous and $Y$ is a Urysohn space, then

$$
A=\left\{\left(x_{1}, x_{2}\right) \mid f\left(x_{1}\right)=f\left(x_{2}\right)\right\}
$$

is $\delta$-preclosed in the product space $X_{1} \times X_{2}$. 
Definition 6. A topological space $X$ is said to be:

(1) $(\delta, p)$-normal if each pair of non-empty disjoint closed sets can be separated by disjoint $\delta$-preopen sets.

(2) Ultra normal [18] if each pair of non-empty disjoint closed sets can be separated by disjoint clopen sets.

Theorem 7. If $f: X \rightarrow Y$ is a contra $\delta$-precontinuous, closed injection and $Y$ is ultra normal, then $X$ is $(\delta, p)$-normal.

Proof. Let $F_{1}$ and $F_{2}$ be disjoint closed subsets of $X$. Since $f$ is closed and injective, $f\left(F_{1}\right)$ and $f\left(F_{2}\right)$ are disjoint closed subsets of $Y$. Since $Y$ is ultra normal $f\left(F_{1}\right)$ and $f\left(F_{2}\right)$ are separated by disjoint clopen sets $V_{1}$ and $V_{2}$, respectively. Hence $F_{i} \subset f^{-1}\left(V_{i}\right), f^{-1}\left(V_{i}\right) \in \delta P O(X, x)$ for $i=1,2$, and

$$
f^{-1}\left(V_{1}\right) \cap f^{-1}\left(V_{2}\right)=\varnothing .
$$

Thus, $X$ is $(\delta, p)$-normal.

\section{REFERENCES}

[1] M. E. Abd El-Monsef, S. N. El-Deeb, and R. A. Mahmoud, " $\beta$-open sets and $\beta$-continuous mapping," Bull. Fac. Sci. Assiut Univ. A, vol. 12, no. 1, pp. 77-90, 1983.

[2] M. Caldas, T. Fukutake, S. Jafari, and T. Noiri, "Some applications of $\delta$-preopen sets in topological spaces," Bull. Inst. Math. Acad. Sinica, vol. 33, no. 3, pp. 261-276, 2005.

[3] M. Caldas and S. Jafari, "Some properties of contra- $\beta$-continuous functions," Mem. Fac. Sci. Kochi Univ. Ser. A Math., vol. 22, pp. 19-28, 2001.

[4] J. Dontchev, "Contra-continuous functions and strongly $S$-closed spaces," Internat. J. Math. Math. Sci., vol. 19, no. 2, pp. 303-310, 1996.

[5] J. Dontchev and T. Noiri, "Contra-semicontinuous functions," Math. Pannon., vol. 10, no. 2, pp. 159-168, 1999.

[6] E. Ekici and T. Noiri, "Contra $\delta$-precontinuous functions," submitted.

[7] T. Fukutake, T. Noiri, M. Caldas, and S. Jafari, "An Alexandroff space defined by $\delta$-preopen sets," Bull. Fukuoka Univ. Ed. III, vol. 54, pp. 1-6, 2005.

[8] S. Jafari and T. Noiri, "Contra-super-continuous functions," Ann. Univ. Sci. Budapest. Eötvös Sect. Math., vol. 42, pp. 27-34 (2000), 1999.

[9] S. Jafari and T. Noiri, "Contra- $\alpha$-continuous functions between topological spaces," Iran. Int. J. Sci., vol. 2, no. 2, pp. 153-167, 2001.

[10] S. Jafari and T. Noiri, "On contra-precontinuous functions," Bull. Malays. Math. Sci. Soc. (2), vol. 25, no. 2, pp. 115-128, 2002.

[11] N. Levine, "Semi-open sets and semi-continuity in topological spaces," Amer. Math. Monthly, vol. 70, pp. 36-41, 1963.

[12] A. S. Mashhour, M. E. Abd El-Monsef, and S. N. El-Deep, "On precontinuous and weak precontinuous mappings," Proc. Math. Phys. Soc. Egypt, no. 53, pp. 47-53 (1983), 1982.

[13] M. Mršević, "On pairwise $R_{0}$ and pairwise $R_{1}$ bitopological spaces," Bull. Math. Soc. Sci. Math. R. S. Roumanie (N.S.), vol. 30(78), no. 2, pp. 141-148, 1986.

[14] O. Njästad, "On some classes of nearly open sets," Pacific J. Math., vol. 15, pp. 961-970, 1965.

[15] S. Raychaudhuri and M. N. Mukherjee, "On $\delta$-almost continuity and $\delta$-preopen sets," Bull. Inst. Math. Acad. Sinica, vol. 21, no. 4, pp. 357-366, 1993. 
[16] S. Raychaudhuri, "Concerning $\delta^{*}$-almost continuity and $\delta$-preregularity," Bull. Calcutta Math. Soc., vol. 85, no. 5, pp. 385-392, 1993.

[17] S. Raychoudhuri and M. N. Mukherjee, " $\delta p$-closedness for topological spaces," J. Indian Acad. Math., vol. 18, no. 1, pp. 89-99, 1996.

[18] R. Staum, "The algebra of bounded continuous functions into a nonarchimedean field," Pacific J. Math., vol. 50, pp. 169-185, 1974.

[19] N. V. Veličko, " $H$-closed topological spaces," Mat. Sb. (N.S.), vol. 70 (112), pp. 98-112, 1966, English transl. in Amer. Math. Soc. Transl., II. Ser., vol. 78, pp. 103-118, 1968.

Authors' addresses

Miguel Caldas

Universidade Federal Fluminense, Departamento de Matematica Aplicada, Rua Mario Santos Braga, s/n, 24020-140, Niteroi, RJ, Brasil

E-mail address: gmamccs@vm.uff.br

\section{Saeid Jafari}

College of Vestsjaelland South, Herrestraede 11, 4200 Slagelse, Denmark

E-mail address: jafariestofanet.dk

Takashi Noiri

Yatsushiro College of Technology, Hirayama shinmachi, Yatsushiro-shi, Kumamoto-ken, 866-8501, Japan

E-mail address: noirieas.yatsushiro-nct.ac.jp

Marilda Simões

Universitá Di Roma "La Sapienza”, Dipartimento Di Matematica "Guido Castelnuovo", Roma, Italia

E-mail address: simoes@mat . uniroma1. it 